\title{
Appareil
}

12 | 2013

Walter Benjamin. Politiques de l'image

\section{Les corps fondamentaux}

\section{Alexandre Costanzo}

\section{(2) OpenEdition}

Journals

Édition électronique

URL : http://journals.openedition.org/appareil/1954

DOI : 10.4000/appareil.1954

ISSN : 2101-0714

\section{Éditeur}

MSH Paris Nord

\section{Référence électronique}

Alexandre Costanzo, «Les corps fondamentaux », Appareil [En ligne], 12 | 2013, mis en ligne le 13 décembre 2013, consulté le 30 juillet 2020. URL : http://journals.openedition.org/appareil/1954 ; DOI : https://doi.org/10.4000/appareil.1954

Ce document a été généré automatiquement le 30 juillet 2020.

\section{(c) (i) () $\Theta$}

Appareil est mis à disposition selon les termes de la Licence Creative Commons Attribution - Pas d'Utilisation Commerciale - Pas de Modification 4.0 International. 


\title{
Les corps fondamentaux
}

\author{
Alexandre Costanzo
}

«il y a des trous, des arrêts, je ne veux pas dire, comprenez-moi bien, dans le temps, je veux dire dans une certaine sorte d'espace ${ }^{1}$ »

Antonin Artaud

\section{Les estropiés à rebours}

1 Dans son diagnostic fameux, Friedrich Nietzsche prévoyait que la civilisation occidentale évoluerait vers ce qu'il appelle le "dernier homme», une créature apathique et infertile, sans grande passion. Incapable de rêver et lassé de l'existence, cet homme ne cherche que le confort et il a découvert la formule du bonheur dans des doses appropriées de petits rêves. Il décrit ainsi une pharmacie où l'épuisement des désirs et des fantasmes se confond à la crudité de la réalité, de sorte qu'entre ses petits rêves et ses jouissances, l'homme occidental aurait égaré quelque part le sens de la vie. Et on trouve dans son Zarathoustra une scène curieuse qui nous plonge dans ce qu'il faudrait caractériser comme à la fois une singulière infirmerie et un étrange paysage :

«Ce m'est pourtant la moindre chose, depuis que je suis parmi les hommes, de voir qu'à l'un il manque un œil, à tel autre une oreille et au troisième une jambe, et que d'aucuns perdirent la langue ou le nez ou la tête. Vois et vis plus vilain, et de telles horreurs que de chacune ne voudrait parler et que d'une d'entre elles je ne saurais me taire, je veux dire : de ces hommes à qui tout manque, sauf qu'une chose ont en excès, - de ces hommes qui rien ne sont qu'un grand œil ou une grande gueule ou un grand ventre, ou n'importe quoi de grand - ceux-là je les nomme des estropiés à rebours.

Et lorsque je sortis de ma solitude et sur ce pont pour la première fois passai, lors je n'en crus pas mes yeux, regardai et de nouveau regardai, et finalement je dis: “Voilà une oreille ! Une oreille aussi grande qu'un homme!" Mieux encore regardai et sous l'oreille remuait encore, en fait, chose pitoyablement petite et souffreteuse et débile. Et en vérité, l'immense oreille tenait sur une petite et frêle tige, - mais cette tige était un homme ! La loupe à l'œil on pouvait même reconnaître encore un visage minuscule et envieux; et qu'à la tige pendait aussi une âme minuscule et boursouflée. Or le peuple me dit que cette grande oreille n'était pas seulement un 
homme, qu'elle était un grand homme, un génie. Mais jamais je ne crus le peuple dans son discours sur les grands hommes - et continuai de croire qu'il s'agit bien d'un estropié à rebours, qui de tout a trop peu et d'une seule chose a trop ".

Lorsque Zarathoustra de la sorte eut au bossu parlé, et à ceux dont il était la bouche et le porte-parole, lors se tourna vers ses disciples, profondément découragé, et dit : "Ô mes amis, en vérité, parmi les hommes je chemine comme parmi des fragments et des morceaux d'homme! L'épouvante de mon œil est que je trouve l'homme morcelé et disjoint comme sur un champ de bataille et qu'équarrissage.

Et si du maintenant mon regard fuit vers le jadis, lors trouve toujours le même spectacle : des fragments, des morceaux, de cruels hasards - mais non des hommes ! Le maintenant et le jadis sur Terre, ah! mes amis, voilà qui m'est le plus intolérable, à moi ! Et je ne pourrais vivre si je n'étais encore un voyant de ce qui nécessairement viendra...2 ${ }^{2}$ ”

2 Nietzsche conçoit une fable où l'on découvre parmi des mendiants et des estropiés, d'étranges infirmes qui seraient autant de grands personnages que compte l'humanité. Au-delà des borgnes, des boiteux, des sourds, des muets, des bossus ou autant de corps mutilés, il nous dit ainsi avoir rencontré avec effroi au détour d'un pont un beau jour un pont, comme ce lieu de passage entre un monde et un autre -, il nous dit donc avoir rencontré une oreille aussi grande qu'un homme, et qu'en y regardant de plus près il se trouve qu'elle tenait sur une petite tige, et que tout cela était en fait un homme. Là une oreille, ici un grand œil, un grand nez ou encore ailleurs un grand ventre, bref des organes et des fragments de corps déliés circulant dans le monde. Mais au-delà du fait d'être monstrueuse, cette grande oreille est avant tout douée d'une puissante capacité : elle entend, et elle entend sans doute mieux que ne le font nos propres oreilles. Tout comme cet œil verra ce que l'on ne voit pas et que ce ventre gigantesque est capable de contenir, de ruminer et de digérer bien des choses... Ce que nous dit Zarathoustra au fond, est qu'il circule entre des organes et des morceaux d'hommes désaccordés, éparpillés, qui sont là comme autant d'énigmes, d'équations erronées, de symptômes errants ou pour le dire autrement, comme des possibilités inachevées. Mais ce champ de bataille avec ses fruits monstrueux germant ici et là comme une pathologie nouvelle, est aussi une sorte de table d'opération: elle porte une promesse avortée de ce qui vient. Et il s'agirait peut-être au fond, tout simplement, d'accoupler ces fragments obscènes, ces organes difformes trouvés au hasard d'un chemin pour inventer ce corps absent en agençant la somme de ces capacités nouvelles, en déplaçant les sens, les significations et les perceptions.

3 Si pour Nietzsche l'homme est quelque chose qu'il faut dépasser, et que le « surhomme » sera le nom de cette forme de vie capable de créer des valeurs nouvelles, ces « estropiés à rebours » désignent la péripétie d'un ratage ou d'une aberration dans le chemin de ce dépassement. Comme c'est le cas dans notre passage, il est souvent question «d'oreilles » dans le poème nietzschéen ${ }^{3}$, conçu comme une grande pièce musicale qui viendrait éveiller une sensibilité nouvelle, une partition avec ses paroles, ses chants et ses danses, car il s'agit avant tout de se faire une oreille pour entendre la voix de l'affirmation. Or cette oreille sur patte ne l'entend pas puisqu'elle va claudicant avec sa lourdeur et il lui manque tout simplement un corps capable de danser. Ceci dit, en s'arrêtant un peu sur ce passage, on dira qu'il nous propose une figure symptomale de la pensée : il est question de la création de "l'homme nouveau » avec des organes appropriés, seul capable de détruire toutes les valeurs sur lesquelles repose l'ordre du monde pour en inventer les nouvelles. Voir, entendre, toucher, parler, sentir, respirer autrement, c'est changer aussi le monde dans lequel nous vivons, c'est le vivre 
autrement. En déplaçant les perceptions, en les accordant ou en désaccordant les sens, pour le dire à la manière d'Arthur Rimbaud, on se fait des yeux et des oreilles nouvelles, c'est-à-dire aussi qu'on se fait un monde nouveau. Et ce que l'on découvre dans l'infirmerie de Nietzsche est en réalité une sorte de musée de la sensibilité dans lequel il devient loisible de déchiffrer une formule singulière du grand rêve d'émancipation de la pensée.

C'est dire que ce lieu nous renvoie à une logique familière dans laquelle on retrouvait déjà les voyances et les voyelles, les hallucinations et le procès d'un « dérèglement de tous les sens " que se proposait Arthur Rimbaud parti en quête de la vraie vie. Et plus tard les corps sans organes, le théâtre de la cruauté ou l'ombilic des limbes d'Antonin Artaud. Cette logique nous dit en somme que le corps manque et que la langue est absente, qu'il n'y a pas encore d'oreille pour entendre, d'œil pour voir ou de bouche pour parler ce monde émancipé - mais qu'on le devine pourtant dans des dérèglements et des intervalles, des affects désaccordés et des possibles avortés - et elle se confond ainsi bien souvent avec le rêve d'un « corps à venir » qu'alimente en quelque sorte un infini plus ou moins obscène.

\title{
2. Le langage de la pensée
}

\author{
«Je mets le doigt sur le point précis de la faille, \\ du glissement inavoué. Car l'esprit est plus \\ reptilien que vous-mêmes, Messieurs, il se dérobe \\ comme les serpents, il se dérobe jusqu'à attenter \\ à nos langues, je veux dire à les laisser en \\ suspens. \\ Je suis celui qui a le mieux senti le désarroi \\ stupéfiant de sa langue dans ses relations avec la \\ pensée ${ }^{4}$. \\ Antonin Artaud
} «Donnez-moi donc un corps ». Par cette expression, Gilles Deleuze caractérise à son tour la formule du renversement philosophique, et il ajoute ceci :

On ne fera plus comparaître la vie devant les catégories de la pensée, on jettera la pensée dans les catégories de la vie. Les catégories de la vie, ce sont précisément les attitudes du corps, ses postures ${ }^{5}$.

6 Le corps n'est plus l'obstacle qui sépare la pensée d'elle-même, ce qu'elle doit surmonter pour arriver à penser mais plutôt ce dans quoi elle plonge pour tendre à l'impensé. Le corps têtu, obstiné, à l'effort. Le corps ivre, fiévreux, sommeillant ou épuisé, comme ce qui force à éprouver ce qui se dérobe à la pensée - la vie. Gilles Deleuze reconfigure donc au détour de cette injonction un paysage embrassant entre autres l'œuvre d'Antonin Artaud que l'on retrouve tout entier dans la tension entre le constat d'une dépossession, d'un " vol des pensées » et cette sorte de quête désespérée : " je suis un homme qui a perdu sa vie et qui cherche par tous les moyens à lui faire reprendre sa place ${ }^{6} »$. Cet homme constate qu'il a perdu sa vie, il constate également qu'il ne pense pas, qu'il ne sait pas parler ou qu'il bégaie parce qu'on mange ses mots, et il va falloir aller chercher ces étranges objets fuyants en commençant par ce qui reste. Et ce qui reste en l'occurrence, aussi instable soit-il, ce qu'il y a, c'est le « corps ». Mais le « corps » envisagé comme " multitude affolée », « une espèce de malle à soufflets qui 
ne peut jamais avoir fini de révéler ce qu'elle recèle. » Le « corps » donc pour que la vie revienne à sa place ou bien pour tenter par tous les moyens de la guérir. Et l'on distinguera, parmi toutes les expériences, ces chemins empruntés : se sentir enfant, arbre, animal, soleil, se confronter à des "pierres parlantes» ou invoquer un corps infini qui serait comme l'ombre de cet autre. Revenir à «tous les vieux moyens éprouvés et magiques de gagner la sensibilité » dans un théâtre de la cruauté. Retrouver le secret d'une langue poétique vivante, rythmée, dansée en partant à la rencontre des Indiens de la Sierra mexicaine, de leurs rites, entre hallucinations et envoûtements, entre des montagnes gravées de signes et un mystérieux alphabet sorti d'un foie pour se répandre dans l'espace. Ou éprouver dans le grouillement immédiat de l'esprit un écheveau de "vibrations", de «forces informulées» pour tenter de donner une voix à ce qu'il caractérisait comme des « larves de pensée ».

7 Le cinéma - comme la littérature ou ce qu'il appelle la "poésie dans l'espace " (le théâtre) - pour Antonin Artaud est d'abord affaire d'ondes neuro-physiologiques produisant ou restituant une sorte de choc nerveux d'où émergera la pensée. Mais on dira avec Deleuze que ce ne sera pas ici sa puissance que le cinéma mettra en avant en proposant la langue nouvelle du monde émancipé plutôt que son essentiel impouvoir. C'est ainsi que dans une lettre fameuse de 1946 à Peter Watson destinée à présenter certains textes, il note : "J'ai débuté dans la littérature en écrivant des poèmes pour dire que je ne pouvais rien écrire du tout, ma pensée quand j'avais quelque chose à dire ou à écrire était ce qui m'était le plus refusé.» Il révèle l'impuissance de la pensée au lieu d'une fêlure, d'une fissure ou d'un refus que manifeste un "on m'a volé mon esprit». L'on pourrait ainsi presque se demander comme une pure question de physique : quel est donc le poids d'une pensée? Car on ne peut plus penser qu'une chose, et c'est que l'on ne pense pas encore. Dans l'embrasure d'un effondrement, d'une dislocation ou d'une pétrification hypnotique que constituerait le choc nerveux des images cinématographiques, c'est la révélation de «l'impouvoir de la pensée » qui force précisément à penser. Cet impouvoir est à la fois source, obstacle, moyen sur lequel il faudra trébucher, d'où la série de scénarii concrets où Artaud ne nous raconte le cinéma qu'à travers ces mêmes histoires de vol, de dépossession ou de fuite ${ }^{7}$ : vampire, fou, suicidé où le " héros est devenu incapable d'accéder à ses pensées ", "réduit à ne voir défiler en lui que des images, un surcroît d'images contradictoires", en proie au vol de son "esprit ${ }^{8}$ ». Il y a d'abord une lézarde, une fissure, des failles comme autant de noyaux de la pensée auxquels viendra se mesurer et se fragmenter tout le reste, telle est la matière que découvre Antonin Artaud. Et Gilles Deleuze le dit exemplairement :

ce n'est plus la pensée qui se confronte au refoulement, à l'inconscient, au rêve, à la sexualité, à la mort [...] ce sont toutes ces déterminations qui se confrontent à la pensée ${ }^{9}$.

Il indique ce faisant le grand renversement auquel procède Artaud, localisant au détour de ce «noyau » un point de butée et de bascule. Aussi il s'agira toujours de compter, de soupeser, d'endurer, de trouver en souffrance des points d'appui pour soulever et supporter ce qui, de la pensée, pèse ${ }^{10}$. Ce que l'on découvre est donc par exemple cet objet insaisissable surgissant dès les premiers textes, le "pèse-nerf ", entendu comme une «station incompréhensible et toute droite au milieu de tout dans l'esprit». Une évaluation du centre de gravité ou du point d'équilibre. Une affaire de poids et de contrepoids, de leviers et poulies, d'attraction et de répulsion, une sorte de balancier pour circonscrire dans une langue une "vibration». Car il s'agit bien d'appréhender ces frémissements, de s'accorder à ce " pouvoir des nerfs de faire durer suffisamment 
une pensée pour que des mots s'y intègrent » et ainsi d'épouser dans un langage ce qui précisément se dérobe.

9 Or la langue d'Artaud est traversée de courts-circuits et de ratages, d'incomplétudes et de lapsus. Si dans un propos fameux, le chorégraphe Merce Cunningham parlait, en regardant les gens dans les rues rattrapant ou étirant par exemple un geste pour ne pas trébucher ou tomber sur un trottoir manqué, de « lapsus de corps », on dira qu'Antonin Artaud construit également sa langue en l'articulant sur des sortes de déséquilibres, des fautes ou des « lapsus » rattrapés. Mais ce qu'il faut rattraper, ce qu'il faut attraper ou construire est une vibration déréglant les langages en se mâchant les mots.

Que se passe-t-il exactement à la lecture de ses écrits? On se trompe bien souvent, on entend un mot pour un autre et un mot par un autre, de sorte que l'on ricoche dans des déséquilibres ou des incertitudes. Tout se passe comme si selon des systèmes d'analogie, de voisinage, de substitution, il employait un mot pour et par un autre concevant tout à la fois des défaillances et des liaisons désaccordées, des battements dont la suite instituerait ainsi la partition d'une vibration. Dans un bel essai, Évelyne Grossman ${ }^{11}$ esquisse le catalogue de ces gestes désorganisant la langue pour ouvrir des fêlures discrètes, agencer des ratés ou des pannes, en faisant déraper l'œil, l'oreille ou la voix de tous ceux qui se confrontent à son œuvre. On trébuche littéralement sur des mots, et tout se passe comme si ces minuscules ratages analphabétiques constituaient des arcs de cercle ou des spirales travaillant de travers, par le dehors, pour aller d'un côté entraver la langue commune, destituer un monde de significations, et partant de l'autre, dans une course effrénée, pour aller rattraper un "quelque chose ", le prendre de vitesse ou en épouser le mouvement: des larves du cerveau, des points de butée de la pensée. Variantes étymologiques, anagrammes imparfaites, glissements en forme de lapsus concertés, phrases décomplétées, proximité de deux mêmes mots ou substitution de l'un pour l'autre, saut d'un idiome à un autre, néologisme posé comme une faute d'orthographe et qui, d'abord inaperçu à la lecture, surgit comme tel pour entrouvrir de petits vides... Tout cela concourt à circonscrire une portée, à creuser des trous et à composer le mouvement d'une vibration qui voudrait s'identifier à celui de la pensée. Si Antonin Artaud est en souffrance de langue, si on lui a "volé », "mangé les mots » et qu'il se trouve confronté à un «mal dire», ce dernier sera précisément l'opérateur d'un dérèglement, d'un suspens ou d'une oscillation pour soupeser ou donner un corps à des larves de pensée surgissant dans des intervalles, des tremblements ou des fuites.

11 On raconte également qu'il martelait souvent un billot dans sa chambre d'Ivry ou qu'il frappait de son couteau les tables des cafés. On pourrait ainsi presque condenser en une image, dans ces gestes, ses mains, l'art d'Antonin Artaud. La percussion - comme l'un des quatre procédés fondamentaux de sa pratique poétique avec la ritournelle, les glossolalies et l'écriture vocale -, la percussion perce le matériau de la langue, fait entendre comme des coups ce qui frappe au "dehors ", des effractions dans l'interstice des mots, des phrases, du langage. Artaud plante inlassablement son couteau ou il frappe à coups de marteau pour malmener et détruire la matière du langage, ponctue, lacère ou martèle la langue pour la faire résonner comme la membrane d'un tambour. Sa main coupe et tranche, et l'on retrouve ainsi cette autre manière de creuser des trous, d'interrompre ou d'inscrire une effraction du « dehors ». Car il n'a jamais écrit, dit-il, que pour « fixer et perpétuer la mémoire de ces coupures, de ces scissions, de ces ruptures, de ces chutes brusques et sans fond qui...» 

La phrase s'interrompt. Il y a un blanc. Un peu plus loin, quatre points de suspension, et puis encore du blanc.

À quoi peut donc ressembler la langue de la pensée ? Comment faire pour en fixer la matérialité ? Tel est finalement le problème d'Antonin Artaud. Mais ce problème est une nécessité, il y va de cette chose perdue qu'est la vie. Ce langage, on le trouve ainsi décrit et construit comme cette langue matérielle, corporelle et vivante que pourrait être la poésie, le dessin, le cinéma ou bien le théâtre. Mais il travaille déjà depuis une autre entrée : ces trous, glissements, failles, suspens. Or il ne s'agit pas de les considérer comme de simples accidents, mais plutôt d'envisager ces déchirures comme le langage même de la pensée. Et c'est de cette "certaine sorte d'espace", comme un empêchement qu'elle se formule. Voilà pourquoi il lui faut ces marteaux et couteaux pour creuser, casser ou entraver, pour ouvrir une sorte d'espace à la langue de la pensée. Voilà aussi pourquoi, il cloue, perce, frappe, élaborant un usage du langage s'articulant sur des trous, des déséquilibres, des glissements ou du blanc. On le disait, la phrase s'interrompt : il y a un blanc. Un peu plus loin, non pas trois mais quatre points de suspension, et puis encore du blanc. Car c'est là où il perd la pensée et le langage, très exactement dans cette sorte de gouffre entre les choses, les mots et le cerveau, ou bien encore dans ce "blanc», qu'une langue s'articule ou articule la pensée, au lieu d'un effondrement.

\section{La constitution des corps}

«Quelle habitude aussi de se percher sur le bord du comptoir et de haranguer de là-haut ses employés! Surtout quand on est dur d'oreille comme le patron et qu'on oblige les gens à s'approcher tout près!».

Franz Kafka

Il y a bien une filiation entre Friedrich Nietzsche et Antonin Artaud, et si elle s'étend jusqu'à l'œuvre de Gilles Deleuze, on pourrait en retracer avec lui la généalogie par cette formule de Spinoza: " nous ne savons pas ce que peut un corps ». Mais ce que peut un corps, est aussi ce à quoi nous confronte le cinéma de Philippe Garrel, là où il éprouve à son tour une sorte de théorème ou plutôt une "physique des corps fondamentaux ${ }^{12} »$.

Dans le noir et le silence le plus complet, une partie du corps et le visage surexposé d'un enfant légèrement de profil se détachent, éclairés par une puissante torche. Il remue et, avec lui, l'ombre portée de son visage sur un mur tout en haut découpant un croissant de lumière ou une sorte d'auréole. Après quelques secondes, des traits lumineux envahissent la pièce par le fond, tout en face de nous. C'est une porte qu'on ouvre et qui reconfigure tout l'espace : un rectangle blanc éblouit le plan. Il y a là un homme qui se tient immobile tandis que la caméra bascule lentement vers le bas. On découvre alors également à contre-jour la silhouette d'une femme assise au premier plan, comme une masse sombre si ce n'est son visage qu'illumine une autre torche en ménageant l'ombre d'une mèche. L'homme derrière elle s'avance et s'assied à ses côtés, il lui donne à boire, lui glisse une cigarette entre les lèvres et enflamme des allumettes. Comme un baiser improbable, ils se relient dans un face à face par des cigarettes mises bout à bout que l'homme incendie finalement d'un même mouvement. Après quelques 
instants, la caméra se redresse et on retrouve le cadre qui ouvrait le plan-séquence, l'enfant perché sur une armoire, cette porte ouvrant sur une profondeur de champ aveuglante : l'homme et la femme se lèvent et marchent pour s'y enfoncer. Il n'y aura eu que des mouvements simples de caméra, vers le bas et puis à nouveau en haut, ouvrant ainsi des "tiroirs" inattendus dans l'espace où s'étageaient les trois personnages entre le premier, le second et l'arrière-plan. Dans la séquence suivante, on retrouve l'homme et la femme dans une singulière chorégraphie descendant un escalier. Cette dernière, cramponnée à la rampe, glisse lentement vers l'avant comme entravée, nouée ou repliée sur elle-même tandis que son compagnon, raide, avance péniblement. Ils émergent cette fois d'un rectangle noir diffus dans le fond de la pièce tout en haut de l'escalier contrastant avec la blancheur clinique des murs et des marches. Et la caméra les attend en contre-plongée jusqu'à ce qu'ils la rejoignent en s'immobilisant - elle courbée au-devant et lui tout droit à l'arrière. Un troisième plan fixe succède où l'on retrouve cette fois assis en tailleur l'enfant, surexposé, il regarde la caméra et semble loger sous ces mêmes escaliers pris par le dessous.

Tel sera le bouleversant prologue du Révélateur (1968) où les deux premiers plans se suivent comme un positif et un négatif ajointés, se renversant en disposant une architecture improbable, et le troisième s'impose comme une variante de l'espace retourné à la manière d'un gant, dans l'atmosphère saturée d'une blancheur laiteuse. Les lieux sont constitués par les jeux du blanc et du noir, par un étagement du plan se recomposant selon que la porte soit fermée ou ouverte, ou selon les déplacements de la caméra faisant surgir un personnage proposant une situation nouvelle. Ils se structurent également suivant des rectangles, des ronds, des triangles ou des quadrilatères découpant des clairs-obscurs se renversant et avec eux notre appréhension: Garrel suspend le temps pour le convertir matériellement en espace s'ouvrant en actes au théâtre d'épisodes psychiques ou pulsionnels. Et on retrouvera ses trois personnages dans des bois, sur les routes, dans des intérieurs, donnant lieu à autant de tableaux de jour ou de nuit. Un enfant, un père et une mère. Du noir et du blanc, du froid et du chaud, des figures géométriques et des séries de postures, ombres, auréoles, éclipses et ellipses, projections : c'est la grammaire cinématographique que propose Philippe Garrel.

L'enfant marche, regarde, attend, rejoint ou est rejoint, il tourne en cercle autour des parents, du lit ou d'un arbre pour « révéler ». Et si la femme court dans les bois la nuit en regardant la caméra, comme pour prendre la mesure, fuir un danger ou maintenir le dialogue, et qu'elle longe très exactement une parallèle, accélérant, elle sera bien souvent plutôt un point fixe, trop fatiguée peut-être, attachée ici et trop lourde ailleurs pour continuer à marcher de sorte qu'elle s'accroche péniblement à son compagnon qui devra finalement la porter sur la route. Ce dernier, quant à lui, tourne autour de luimême ou décrit des arcs de cercle et des tangentes, se tient souvent à l'arrière du tableau qu'il compose avec la femme et l'enfant, le traverse pour y entrer pleinement et assumer des rapports de famille. Il donne à boire et à fumer, il soulève, rejoint, devance ou accompagne. C'est ainsi que selon des suites de figures géométriques et de postures des corps, ils se tournent autour, éclatent ou se retrouvent. Un tableau s'ouvre avec l'enfant généralement qui sert de boussole à la caméra, donne lieu à des figures, propose des directions et des situations et l'on retourne finalement à ce point d'ancrage mouvant qu'est l'enfant. 
Debout, assis, couché, se lever, sauter, ramper, marcher, courir, caresser, étreindre, enlacer. Hochements de tête, tremblements, immobilités, lassitudes, inquiétudes, prières, fuites et poursuites... C'est un catalogue de gestes, d'attitudes et de postures qui se succèdent, saturé par des paroles à blanc, nourri de mimiques, de jeux et de scènes de ménage à un, à deux et à trois, suivant les combinaisons. Et tout semble se passer " avant ", "après » ou bien alors "pendant ». Philippe Garrel décrit ainsi les scènes primordiales d'une humanité générique. Elles sont d'autant plus épurées et viscérales qu'elles baignent dans la pathologie familiale, l'onirisme ou les cauchemars qu'intensifient le noir et les flashs, les fondus, la blancheur laiteuse et les ombres portées, la geste insensée ou les décors désertés : route, forêt, trous, fondrières, rivière, théâtre, maison... Et on retrouve toujours ces mêmes figures: cercles et courbes, parallèles et droites asymptotiques ou convergentes, auréoles, rectangles, quadrilatères et toupies. C'est presque un traité de géométrie et d'architecture que compose Garrel mais uniquement en tant qu'il tient aux corps, à leur pesanteur propre. Point d'ancrage assis, couché ou mobile, liant et reliant. Point fixe érotique, sombre ou lourd. Longer, accompagner, porter et tourner sur soi-même. Ce sont les trois "positions" qu'assument respectivement, l'enfant, la mère et le père, les éléments d'un alphabet primordial qui donnera lieu à des combinatoires pour constituer ou épuiser un répertoire. C'est ainsi que le garçon rejoindra sur la route ses parents agenouillés, en prière devant un lit, et qu'il les encerclera à plusieurs reprises. Ou bien plus tard, comme une variante et toujours après un trajet de nuit, il les retrouve couchés dans le petit lit blanc posé à même le sol. Ils s'y allongeront alternativement comme dans une ronde où chacun étreindra un instant l'un des deux autres avant de repartir. Et tout s'articulera autour de l'enfant: les mouvements des parents aussi bien que ceux de la caméra polarisée sur ce point d'ancrage mobile ouvrant et refermant le film, ainsi que la plupart des séquences.

Philippe Garrel propose alors, comme on dit, un " cinéma de la révélation ». Le cinéma révélant et révélé au détour de cérémonies, d'une liturgie, d'un théâtre : une physique des corps. Sur fond d'une séparation, d'un désaccord, d'une cicatrice, ses personnages seront alors la femme, l'homme et l'enfant, trinité répondant à celle des acteurs, du cinéaste et de la camér $\mathrm{a}^{13}$, ou configurant avec cette dernière une sorte de quadrilatère. Mais tout se passe bien « avant » dans un temps de l'origine avec le feu, la glace, l'eau, la terre, le vent, des voix, comme éléments premiers constituants (La cicatrice intérieure), " après » ou bien alors " pendant »- dans l'espace angoissé du rêve ou dans l'à-côté d'une parenthèse. C'est depuis le noir et le blanc que surgissent les corps pour se redéployer avec les architectures, sans que l'on puisse jamais comprendre dans quel espace nous nous situons (Le révélateur, La concentration). Et c'est en fonction du chaud et du froid, des déserts de sable ou de glace, que s'ajusteront des ensembles de postures et d'attitudes : on a froid et on a chaud avec les films de Garrel, que ce soit induit par le climat, la nuit, le jour, les projections lumineuses ou bien le goût glacé de la drogue, de l'hôpital ou de la mort (de L'Enfant secret à Un été brûlant en passant par Sauvage innocence). On a chaud et on a froid surtout, c'est dire que nous faisons corps avec une physique élémentaire, aussi bien qu'avec la sensualité des mouvements de la caméra.

Si Philippe Garrel compose un théâtre de gestes, la caméra épousera quant à elle des déplacements correspondant à ce répertoire en décrivant à son tour des cercles, des lignes, des mouvements de bascule pour accompagner ou retrouver, suivant des ellipses, les corps. Il y aura ainsi une séquence où la caméra quitte les personnages du 
Révélateur frappant à la porte d'une maison dans laquelle ils comptaient se réfugier après avoir fui on ne sait quelle menace. Elle les abandonne par un travelling longeant parallèlement la demeure pour tourner ensuite sur elle-même en décrivant une courbe et en prenant au passage la mesure de l'horizon, une large crevasse, dans un panoramique étourdissant, jusqu'à ce qu'elle s'arrête un bref instant en retrouvant de manière inattendue les trois personnages. La mère confiera alors l'enfant au père qui le portera pour le déposer sur un pneu en contrebas. Tandis qu'ils avancent ainsi tous trois, la caméra les fixe en suivant un parcours latéral puis une nouvelle courbe jusqu'à son point d'arrivée où elle attend - après avoir donc dessiné dans l'espace une sorte de $\mathrm{S}$. L'homme et la femme escaladant la carrière par une autre entrée surgissent alors par le dessous pour se fixer exactement en face de l'opérateur en serrant, moyennant quelques convulsions, un fil barbelé. Si la caméra redouble le mouvement de l'homme au début du film tournant sur lui-même comme une toupie, si elle recourt à une ronde ou ailleurs à de longues boucles, si elle rampe à son tour et plonge dans les hautes herbes convoquée par un mouvement de main de l'enfant à la suite des personnages, c'est toujours pour rejoindre en un point précis les corps, pour s'accorder à la dramaturgie. Tout se passe ainsi comme si elle était soumise à une attraction ou bien à une mathématique rigoureuse, prise dans son propre mouvement répondant à des lois géométriques et physiques pour croiser selon une familiarité cosmologique ${ }^{14}$ les corps fondamentaux. Elle s'ordonne ou s'aligne, comme on le dirait de planètes, pour construire au détour d'ellipses la situation la plus favorable.

On aura souvent évoqué le travelling en U dans La concentration (1968) ou bien Joseph tournant en regardant la caméra qui change elle-même successivement de position dans Marie pour mémoire (1967). Mais c'est dans La cicatrice intérieure (1971) que l'on retrouvera cette grammaire dans sa forme la plus épurée. Ici encore la femme (Nico) est d'abord assignable à un point fixe pesant et on la découvre ainsi dans le prologue assise, épuisée, criant et larmoyante, tandis que son compagnon (Philippe Garrel) est en mouvement : il marche, soutient, accompagne, devance. Et dans un plan fameux, Garrel se tenant debout auprès d'elle - juchée sur le sol en composant une pyramide -, lâche sa main pour partir en marchant suivant une ligne droite. Or quel n'est pas notre étonnement en constatant que dans un glissement imperceptible cette ligne latérale se fait cercle dès lors qu'il la rejoint et l'enjambe pour réitérer plusieurs révolutions. Si le travelling circulaire coordonné sur la marche nous expose à un espace impossible en dépravant la perspective, il révèle avant tout une dramaturgie de gestes élémentaires en les inscrivant dans le tracé de figures géométriques corrompues.

La caméra s'emportera ainsi dans des chorégraphies simples ou complexes, plans fixes basculant légèrement, zoom avant ou arrière, travelling latéraux, circulaires et ailleurs en $S$ ou en $U$, pour découvrir des situations nouvelles dans un même panneau savamment étagé où l'on retrouvera en des points circonscrits la partition des personnages. On ne sait ainsi jamais où nous emmène un plan-séquence qui semble se poursuivre après les fondus, et nous sommes toujours pris entre deux mouvements autonomes: ceux de la caméra que nous épousons se coordonnant selon une gravitation énigmatique à ceux des personnages qui vont à pied, à cheval, en bateau, s'arrêtent, traversent ou disparaissent dans les lacets de la profondeur de champ. La grammaire cinématographique emportée par ses propres nécessités, fondamentalement désaccordée pour accomplir ses ellipses, se coordonne en répondant au langage des corps (actes, postures, attitudes, voix, silences), de sorte que nous glissons tout le long du film, emportés par des déplacements, embarqués dans des 
sortes de véhicules, pour accompagner une geste. De cette dernière, on dira qu'elle se fixe en des formes symboliques premières, qu'elle résulte d'une réduction à des langages élémentaires ou bien qu'elle assume dans sa trivialité le théâtre pathologique de la gesticulation humaine. Mais tout cela renvoie finalement à la naissance d'un monde ou du monde. Car le problème est sans doute le suivant : comment constituer, renouveler, comment changer le monde ? Pour ce faire, on confiera à un enfant la tâche de le révéler. On peut aussi le dire trivialement en se basant sur ce qu'est le sens biologique de l'existence : quand on ne sait plus comment faire pour changer le monde, alors on fait un enfant en espérant qu'il lui reviendra d'affirmer - aussi un peu pour nous ${ }^{15}$ - le possible. Il est d'ailleurs remarquable que lors d'un entretien en $1968^{16}$, Philippe Garrel décrivait ses films comme autant d'avortons : il les fabrique à défaut d'avoir fait un enfant. Mais ce que je veux dire, c'est qu'il renouvelle profondément ou invente alors dans le cinéma le personnage de l'enfant. Et voilà que ce dernier encercle les parents autour du petit lit aux draps blancs comme pour percer le mystère de ses origines, se recueille aussi bien sur ce morceau d'écran autour duquel il tourne et travaille ainsi à révéler comme une promesse le monde. S'il est proche en tout ceci de la tradition burlesque, je dirais volontiers que Philippe Garrel propose alors un cinéma d'enfant, pour enfant, pour l'enfant.

Le cinéma de Philippe Garrel est bien affaire de constitution des corps et participe en ce sens d'un compagnonnage des œuvres de Friedrich Nietzsche, Antonin Artaud ou encore Franz Kafka. Si le premier décrivait un grand ballet pour inventer un corps capable de danser, que le second localise un corps infini, un gouffre ou une certaine sorte d'espace articulant le langage de la pensée, il revient à Kafka d'avoir envisagé le lieu où l'humanité se reconnaîtrait enfin pleinement émancipée. C'est ce qu'il désignait comme le théâtre d'Oklahoma ${ }^{17}$ où les acteurs engagés n'auraient rien d'autre à faire que d'être fidèles à leurs propres gestes, ici et maintenant. C'est aussi bien ce qui explique toutes les postures incongrues sillonnant ses œuvres, entre des têtes penchées, cet homme incapable de se remettre "sur le côté » pour dormir ou encore cette habitude de son patron de se "percher sur le bord du comptoir ", interpellant de là-haut ses employés d'autant que, dur d'oreille, il oblige les gens «à s'approcher tout près $^{18} »$. Si on peut envisager les livres de Kafka comme un immense répertoire de postures improbables dans un monde mal en ordre, la gesticulation de ses personnages n'est rien d'autre qu'une tentative désespérée pour remettre précisément les choses ou le monde en place. Mais tout se passe comme s'ils n'effectuaient pas ces gestes au bon moment ou au bon endroit et il n'y aurait guère que ce lieu, le théâtre d'Oklahoma où l'on serait en quelque sorte enfin fidèle à ses actes et à son existence. Peut-être que les œuvres de Philippe Garrel ne font à leur tour rien d'autre que de constituer un alphabet de gestes, de voix et de situations élémentaires d'une humanité générique, proposant ainsi une genèse ou reconstituant sans arrêt le corpus des acquis.

Ce langage remonte sans doute depuis ou avec le cinéma muet, mais il repose avant tout sur l'adéquation entre une grammaire, des suites de figures géométriques convergeant avec des gestes premiers. Mais que peuvent bien signifier ces chorégraphies, agencements triangulaires ou ce quadrilatère liant les personnages à la caméra? Et puis ces rondes, ces boucles, ces révolutions? Ce que l'on découvre avec Garrel est sans doute une autre tentative désespérée de consigner une puissance, à savoir ce que peut en définitive un corps qu'il s'attache à encercler. Les révolutions induisent certainement un enfermement, un abrutissement où l'on tourne en rond avant de repartir ailleurs désorienté. Mais c'est une manière aussi de garder, d'entourer 
le possible pour le préserver. Il dépose ainsi des formes et des forces élémentaires, un alphabet génétique, eschatologique et révolutionnaire, où l'on ne reconnaît finalement rien d'autre que ce que peut, dans sa trivialité, un homme.

\section{BIBLIOGRAPHIE}

Benjamin Walter, Euvres II, Maurice de Gandillac, Pierre Rusch et Rainer Rochlitz (trad.), Paris, Gallimard, 2000.

Deleuze Gilles, L'image-temps, cinéma 2, Paris, Minuit, 1994.

Deleuze Gilles, « Le mystère d'Ariane selon Nietzsche », in Gilles Deleuze, Critique et clinique, Paris, Minuit, 1993.

Garrel Philippe, «Cerclé sous vide », entretien avec Jean-Louis Comolli, Jean Narboni et Jacques Rivette, Cahiers du cinéma, $\mathrm{n}^{\circ} 204$, septembre 1968.

Grossman Evelyne, Artaud, l'aliéné authentique, Paris, Farrago/Éditions Léo Scheer, 2003.

Kafka Franz, L’Amérique, Paris, Gallimard (Folio), 1998.

Kafka Franz, La Métamorphose, Paris, Gallimard (Folio), 1995.

Nancy Jean-Luc, Corpus, Paris, Métailié, 1992.

Philippon Alain, « L'enfant-cinéma », Cahiers du cinéma, nº 344, 1983.

\section{NOTES}

1. Antonin Artaud, L'ombilic des limbes, Paris, Gallimard, 1994.

2. Friedrich Nietzsche, Ainsi parlait Zarathoustra, Maurice de Gandillac (trad.), Paris, Gallimard (Folio), 1989.

3. Voir Gilles Deleuze, «Le mystère d'Ariane selon Nietzsche », in Critique et clinique, Paris, Minuit, 1993.

4. Antonin Artaud, L'ombilic des limbes.

5. Gilles Deleuze, L'image-temps, cinéma 2, Paris, Minuit, 1994, p. 246.

6. Ibid., p. 225.

7. Ibid., p. 217. Deleuze évoque respectivement « 32 », «La révolte des bouchers ", « Dix-huit secondes » en ce qui concerne le "vol des pensées », et plus loin «La coquille et le clergyman » pour illustrer le traitement diurne qu'Artaud fait subir au rêve renversant ainsi la figure du somnambule expressionniste.

8. Ibid., p. 217.

9. Ibid., p. 217.

10. Voir Évelyne Grossman, Artaud, l'aliéné authentique, Paris, Farrago/Éditions Léo Scheer, 2003, p. 166. Et en ce qui concerne l'articulation entre pesée et pensée : Jean-Luc Nancy, Corpus, Paris, Métailié, 1992.

11. Évelyne Grossman, Artaud, l'aliéné authentique, p. 63-97.

12. Gilles Deleuze, L'image-temps, cinéma 2, p. 258. 
13. Ibid., p. 262-263.

14. Alain Philippon, « L'enfant-cinéma », Cahiers du cinéma, $\mathrm{n}^{\circ} 344,1983$.

15. Je renvoie aux passages fameux de Walter Benjamin dans ses «Thèses sur la philosophie de l'histoire » convoquant cette « faible force » messianique.

16. Cf. «Cerclé sous vide », entretien avec Philippe Garrel par Jean-Louis Comolli, Jean Narboni et Jacques Rivette, Cahiers du cinéma, nº 204, septembre 1968.

17. Franz Kafka, L'Amérique, Paris, Gallimard (Folio), 1998. Je pense naturellement ici aux essais qu'aura consacrés Walter Benjamin à Kafka in CEuvres II, Paris, Gallimard, 2000.

18. Franz Kafka, La Métamorphose, Paris, Gallimard (Folio), 1995.

\section{RÉSUMÉS}

Comme un compagnonnage de l'œuvre de Benjamin et de ce qu'il en est du statut des gestes dans son œuvre, je prendrai comme fil conducteur Gilles Deleuze pour confronter les pensées de Friedrich Nietzsche, Antonin Artaud et les premiers films de Philippe Garrel, en tentant de reformuler à travers elles ce qu'il définit comme puissance ou impuissance des " corps ». Mais, ce faisant, mon idée est de circonscrire à travers ces œuvres autant de paysages affirmant des pensées de l'émancipation.

INDEX

Mots-clés : émancipation, corps

Personnes citées : Artaud (Antonin), Garrel (Philippe), Nietzsche (Friedrich)

\section{AUTEUR}

\section{ALEXANDRE COSTANZO}

Alexandre Costanzo est professeur à l'École d'art d'Annecy et travaille à une thèse de philosophie portant sur le thème de l'émancipation, sous la direction d'Alain Badiou. 